\title{
ON VECTORIAL NORMS AND PSEUDONORMS
}

\section{EMERIC DEUTSCH}

AbSTRACT. A vectorial pseudonorm (norm) of order $k$ on the vector space $C^{n}$ of all $n$-tuples of complex numbers is a mapping from $C^{n}$ into the positive cone of $R^{k}$ which satisfies the usual axioms of a pseudonorm (norm). The vector space $R^{k}$ of the $k$-tuples of real numbers is partially ordered componentwise. Vectorial norms have been introduced by Kantorovitch. Recently they have been studied by Robert and Stoer. In the present paper different properties of vectorial pseudonorms are investigated. They deal mainly with the following topics: regularity of pseudonorms, the dual of a vectorial norm, inequality between vectorial pseudonorms and the $G$-transform of a vectorial pseudonorm.

1. Introduction. Let $C^{n}$ denote the vector space of all $n$-tuples of complex numbers, and let $R_{+}^{\mathbf{k}}$ denote the set of all $k$-tuples of nonnegative real numbers partially ordered componentwise. A vectorial norm of order $k$ on $C^{n}$ is a mapping $p: C^{n}>R_{+}^{k}$ such that

$$
\begin{aligned}
p(\alpha x) & =|\alpha| p(x), & & \forall x \in C^{n}, \quad \forall \alpha \in C, \\
p(x+y) & \leqq p(x)+p(y), & & \forall x, y \in C^{n}, \\
p(x) & \neq 0 \quad \text { if } x \neq 0 . & &
\end{aligned}
$$

Vectorial norms have been introduced by Kantorovitch [4]. Recently they have been studied by Robert [9], [10], [11] and by Stoer [12]. Special types of vectorial norms were studied by Kantorovitch, Vulikh and Pinsker [5], [6], Ostrowski [7], [8], Fiedler and Pták [1].

A mapping $p: C^{n} \rightarrow R_{+}^{k}$ which satisfies axioms (1.1) and (1.2) will be called a vectorial pseudonorm of order $k$ on $C^{n}$. We will denote by $p_{1}(x), \cdots, p_{k}(x)$ the components of $p(x)$. It is clear that $p$ is a vectorial pseudonorm if and only if the mapping $x \rightarrow p_{j}(x)\left(x \in C^{n}\right)$ is a pseudonorm on $C^{n}$ for each $j=1, \cdots, k$.

To every vectorial pseudonorm $p: C^{n} \rightarrow R_{+}^{k}$ we associate the following subspaces of $C^{n}$ :

$$
\begin{aligned}
K_{j}(p) & =\left\{x \in C^{n}: p_{j}(x)=0\right\} \quad(j=1, \cdots, k), \\
K(p) & =\bigcap_{h} K_{h}(p),
\end{aligned}
$$

Received by the editors March 30, 1970.

AMS 1969 subject classifications. Primary 1505, 4610.

Key words and phrases. Vectorial norm, vectorial pseudonorm, regular vectorial norm, dual of a vectorial norm. 


$$
\begin{aligned}
W_{j}(p) & =\bigcap_{h \neq j} K_{h}(p) \quad(j=1, \cdots, k), \\
W(p) & =W_{1}(p)+\cdots+W_{k}(p) .
\end{aligned}
$$

Properties of these subspaces for the case of a vectorial norm $p$, can be found in [9] and [10]. For example, $p$ is a vectorial norm if and only if $K(p)=\{0\}$. It is easy to see that for an arbitrary vectorial pseudonorm $p$, we have

$$
\begin{aligned}
\sum_{h \neq j} W_{h}(p) & \subseteq K_{j}(p) & (j=1, \cdots, k), \\
W(p) & \subseteq K_{j}(p)+W_{j}(p) & (j=1, \cdots, k) .
\end{aligned}
$$

Two vectorial pseudonorms $p, q: C^{n} \rightarrow R_{+}$will be called congruent if $W_{j}(p)=W_{j}(q)$ for all $j=1, \cdots, k$.

2. Regular vectorial pseudonorms. A vectorial pseudonorm $p: C^{n} \rightarrow R_{+}^{k}$ is said to be regular if $W(p)=C^{n}$.

ExAmple 1. Consider the mapping $p: C^{3} \rightarrow R_{+}^{2}, p(\alpha, \beta, \gamma)$ $=(|\alpha|,|\gamma|)$. We have

$$
\begin{aligned}
& K_{1}(p)=W_{2}(p)=\{(0, \beta, \gamma): \beta, \gamma \in C\}, \\
& K_{2}(p)=W_{1}(p)=\{(\alpha, \beta, 0): \alpha, \beta \in C\} .
\end{aligned}
$$

Thus $W_{1}(p)+W_{2}(p)=C^{3}$ and so $p$ is a regular vectorial pseudonorm. Note that $p$ is not a vectorial norm.

Proposition 1. If $p: C^{n} \rightarrow R_{+}^{k}$ is a vectorial norm, then the following statements are equivalent:

(i) $p$ is regular;

(ii) there exists a norm $\nu$ on $C^{n}$ and a direct-sum decomposition $C^{n}=X_{1} \oplus \cdots \oplus X_{k}$ with associated projections $E_{1}, \cdots, E_{k}$ such that

$$
p(x)=\left(\nu\left(E_{1} x\right), \cdots, \nu\left(E_{k} x\right)\right), \quad \forall x \in C^{n} ;
$$

(iii) if $p(x)=u+v, u, v \in R_{+}^{k}$, then there exist vectors $y_{s} z \in C^{n}$ such that $x=y+z, p(y)=u$, and $p(z)=v$;

(iv) $C^{n}=K_{j}(p) \oplus W_{j}(p), \forall j=1, \cdots, k$.

Proof. (i) $\Rightarrow$ (ii). It is known [10] that the subspaces $W_{1}(p), \cdots$, $W_{k}(p)$ are independent which together with the regularity of $p$ shows that $C^{n}=W_{1}(p) \oplus \cdots \oplus W_{k}(p)$. Let $E_{1}, \cdots, E_{k}$ be the projections associated with this direct-sum decomposition. It is easy to see that the mapping $x \rightarrow \nu(x)=\operatorname{Max}_{h} p_{h}(x)$ is a norm on $C^{n}$. Since $p_{i}(x)$ $=p_{i}\left(E_{i} x\right)[10]$, we have, for a!l $j=1, \cdots, k$ and for all $x \in C^{n}$,

$$
\nu\left(E_{j} x\right)=\operatorname{Max}_{h}\left\{p_{h}\left(E_{j} x\right)\right\}=\operatorname{Max}_{h}\left\{p_{h}\left(E_{h} E_{j} x\right)\right\}=p_{j}\left(E_{j} x\right)=p_{j}(x) .
$$


(ii) $\Rightarrow$ (iii). The proof of this implication can be found in [1].

(iii) $\Rightarrow$ (iv). Let $x \in C^{n}$ and let $j \in\{1, \cdots, k\}$. Denote

$$
u=\left(p_{1}(x), \cdots, p_{j-1}(x), 0, p_{j+1}(x), \cdots, p_{k}(x)\right), \quad v=p(x)-u .
$$

Then $p(x)=u+v$ and by assumption there exist vectors $y, z \in C^{n}$ such that $x=y+z, p(y)=u$, and $p(z)=v$. Then $p_{j}(y)=0$ and $p_{h}(z)=0$ for $h \neq j$. Hence $y \in K_{j}(p), z \in W_{j}(p)$ and thus $x=y+z \in K_{j}(p)+W_{j}(p)$. Therefore $C^{n}=K_{j}(p)+W_{j}(p)$. It can be easily seen that $K_{j}(p)$ $\cap W_{j}(p)=\{0\}$.

(iv) $\Rightarrow(\mathrm{i})$. This implication is known [10].

Remark 1. Kantorovitch, Vulikh and Pinsker [5], [6] consider mappings which satisfy axioms (1.1)-(1.3) and property (iii) of Proposition 1. Fiedler and Pták [1] consider mappings which are obtained through the procedure indicated in property (ii) of Proposition 1. Consequently, Proposition 1 shows that all these mappings are exactly the regular vectorial norms.

PROPOSITION 2. If $p: C^{n} \rightarrow R_{+}^{k}$ is a regular vectorial norm, then

$$
K_{j}(p)=\sum_{h \neq j} \oplus W_{h}(p) \quad(j=1, \cdots, k) .
$$

Proof. Let $x \in K_{j}(p)$. Then $p_{j}(x)=0$ and since $p$ is regular we have $x=x_{1}+\cdots+x_{k}$ with $x_{h} \in W_{h}(p) \quad(h=1, \cdots, k)$. Now $p_{j}\left(x_{j}\right)=p_{j}(x)$ $=0$ whence $x_{j}=0$ (see [10]). Then

$$
x=x_{1}+\cdots+x_{j-1}+x_{j+1}+\cdots+x_{k} \in \sum_{h \neq j} W_{h}(p) .
$$

Thus $K_{j}(p) \subseteq \sum_{h \neq j} W_{h}(p)$, which together with (1.4) and with the fact that the $W_{h}(p)$ 's are independent proves our claim.

Remark 2. The converse of Proposition 2 is not true. Consider for example the vectorial norm $p: C^{4} \rightarrow R_{+}^{3}$ defined by

$$
p\left(\alpha_{1}, \alpha_{2}, \alpha_{3}, \alpha_{4}\right)=\left(\left|\alpha_{1}\right|+\left|\alpha_{4}\right|,\left|\alpha_{2}\right|+\left|\alpha_{4}\right|,\left|\alpha_{3}\right|+\left|\alpha_{4}\right|\right) \text {. }
$$

We have

$$
\begin{aligned}
& K_{1}(p)=\left\{\left(0, \alpha_{2}, \alpha_{3}, 0\right): \alpha_{2}, \alpha_{3} \in C\right\}, \\
& K_{2}(p)=\left\{\left(\alpha_{1}, 0, \alpha_{3}, 0\right): \alpha_{1}, \alpha_{3} \in C\right\}, \\
& K_{3}(p)=\left\{\left(\alpha_{1}, \alpha_{2}, 0,0\right): \alpha_{1}, \alpha_{2} \in C\right\}, \\
& W_{1}(p)=\left\{\left(\alpha_{1}, 0,0,0\right): \alpha_{1} \in C\right\}, \\
& W_{2}(p)=\left\{\left(0, \alpha_{2}, 0,0\right): \alpha_{2} \in C\right\}, \\
& W_{3}(p)=\left\{\left(0,0, \alpha_{3}, 0\right): \alpha_{3} \in C\right\} .
\end{aligned}
$$


Thus $K_{j}(p)=\sum_{h \neq j} \oplus W_{h}(p)(j=1,2,3)$ but $p$ is not regular since $\sum_{h} W_{h}(p) \neq C^{4}$.

Proposition 3. Let $p$ and $q$ be vectorial pseudonorms of order $k$ on $C^{n}$ and assume that $p \leqq q\left(i . e . p(x) \leqq q(x), \forall x \in C^{n}\right)$.

(a) If $p$ is a vectorial norm, then $q$ is a vectorial norm.

(b) If $q$ is regular, then $p$ is regular.

ProOF. It can be easily shown that $p \leqq q$ implies

$$
\begin{array}{rlrl}
K_{j}(q) & \subseteq K_{j}(p), & W_{j}(q) \subseteq W_{j}(p) & (j=1, \cdots, k), \\
K(q) \subseteq K(p), & W(q) \subseteq W(p) . &
\end{array}
$$

From relations (2.2) we obtain both statements (a) and (b).

Proposition 4. Let $p$ and $q$ be regular vectorial norms of order $k$ on $C^{n}$ such that $p \leqq q$. Then

(a) $K_{j}(p)=K_{j}(q)(j=1, \cdots, k)$;

(b) $W_{j}(p)=W_{j}(q)(j=1, \cdots, k)$, (i.e. $p$ and $q$ are congruent).

PROOF. Since $p$ and $q$ are both regular, we have for all $j=1, \cdots, k$ (see Proposition 1)

$$
K_{j}(p) \oplus W_{j}(p)=K_{j}(q) \oplus W_{j}(q)=C^{n} .
$$

Now relations (2.1) together with a dimension argument will give $K_{j}(p)=K_{j}(q)$ and $W_{j}(p)=W_{j}(q)$ for all $j=1, \cdots, k$.

3. The dual of a vectorial norm. Let $p: C^{n} \rightarrow R_{+}^{\mathrm{k}}$ be a vectorial norm and consider the mappings

$$
q_{j}: C^{n} \rightarrow R, \quad q_{j}(y)=\operatorname{Sup}_{x \in W_{j}(p) ; x \neq 0} \frac{\left|y^{*} x\right|}{p_{j}(x)} \quad\left(y \in C^{n}\right)
$$

for each $j=1, \cdots, k$. Then it is easy to see that the mapping

$$
p^{D}: C^{n} \rightarrow R_{+}^{k}, \quad p^{D}(y)=\left(q_{1}(y), \cdots, q_{k}(y)\right) \quad\left(y \in C^{n}\right)
$$

is a vectorial pseudonorm of order $k$ on $C^{n}$. This mapping $p^{D}$ will be called the dual of $p$. In [10] the dual has been defined only for regular vectorial norms.

Proposition 5. If $p$ is a vectorial norm, then
(a) $K_{j}\left(p^{D}\right)=\left(W_{j}(p)\right)^{\perp}(j=1, \cdots, k)$,
(b) $W_{j}\left(p^{D}\right) \supseteq\left(K_{j}(p)\right)^{\perp}(j=1, \cdots, k)$,
(c) $K\left(p^{D}\right)=(W(p))^{\perp}$,
(d) $p^{D}$ is regular. 
Proof. (a) $K_{\jmath}\left(p^{D}\right)=\left\{y \in C^{n}: g_{\jmath}(y)=0\right\}=\left\{y \in C^{n}: y^{*} x=0\right.$, $\left.\forall x \in W_{j}(p)\right\}=\left(W_{j}(p)\right)^{\perp}$;

(b) $W_{j}\left(p^{D}\right)=\bigcap_{h \neq j} K_{h}\left(p^{D}\right)=\bigcap_{h \neq j}\left(W_{h}(p)\right)^{\perp}=\left(\sum_{h \neq j} W_{h}(p)\right)^{\perp} \supseteq$ $\left(K_{,}(p)\right)^{\perp}$, where we have made use of relation $(1.4)$;

(c) $K\left(p^{D}\right)=\bigcap_{h} K_{h}\left(p^{D}\right)=\bigcap_{h}\left(W_{h}(p)\right)^{\perp}=\left(\sum_{h} W_{h}(p)\right)^{\perp}=(W(p))^{\perp}$;

(d) $W\left(p^{D}\right)=\sum_{h} W_{h}\left(p^{D}\right) \supseteq \sum_{h}\left(K_{h}(p)\right)^{\perp}=\left(\bigcap_{h} K_{h}(p)\right)^{\perp}=C^{n}$, i.e. $p^{D}$ is regular.

As an immediate consequence of relation (c) we have the following

COROLLARY. $p^{D}$ is a vectorial norm if and only if $p$ is regular.

Proposition 6. If $p$ is a regular vectorial norm, then $W_{j}\left(p^{D}\right)$ $=\left(K_{j}(p)\right)^{\perp}$.

Proof. Making use of Propositions 2 and 5, we have

$$
\begin{aligned}
W_{j}\left(p_{D}\right) \supseteq\left(K_{j}(p)\right)^{\perp} & =\left(\sum_{h \neq j} W_{h}(p)\right)^{\perp}=\bigcap_{h \neq j}\left(W_{h}(p)\right)^{\perp}=\bigcap_{h \neq j} K_{h}\left(p^{D}\right) \\
& =W_{j}\left(p^{D}\right) .
\end{aligned}
$$

Proposition 7. If $p, q: C^{n} \rightarrow R_{+}^{\mathbf{k}}$ are vectorial norms such that $p \leqq q$, then $q^{D} \leqq p^{D}$.

Proof. Making use of $(2.1)$, we have, for all $j=1, \cdots, k$ and all $y \in C^{n}$,

$$
p_{j}^{D}(y)=\operatorname{Sup}_{x \in W_{j}(p) ; x \neq 0} \frac{\left|y^{*} x\right|}{p_{j}(x)} \geqq \operatorname{Sup}_{x \in W_{j}(q) ; x \neq 0} \frac{\left|y^{*} x\right|}{q_{j}(x)}=q_{j}^{D}(y) .
$$

PROPOSITION 8. If $p$ is a regular vectorial norm of order $k$ on $C^{n}$ such that $W_{i}(p) \perp W_{j}(p)$ for $i \neq j$, then

(a) $K_{j}\left(p^{D}\right)=K_{j}(p)(j=1, \cdots, k)$;

(b) $W_{j}\left(p^{D}\right)=W_{j}(p)(j=1, \cdots, k)$ i.e. $p$ and $p^{D}$ are congruent.

Proof. From Proposition 2 it follows that $W_{j}(p) \perp K_{j}(p)$ for all $j=1, \cdots, k$. Since $W_{j}(p) \oplus K_{j}(p)=C^{n}$ (see Proposition 1) we obtain, from Proposition $5(\mathrm{a}), K_{j}\left(p^{D}\right)=\left(W_{j}(p)\right)^{\perp}=K_{j}(p)(j=1, \cdots, k)$, and similarly, from Proposition 6, $W_{j}\left(p^{D}\right)=\left(K_{j}(p)\right)^{\perp}=W_{j}(p)$ $(j=1, \cdots, k)$.

REMARK 3. In particular, statements (a) and (b) of Proposition 8 hold if $p$ is obtained from an orthogonal decomposition of $C^{n}$ and a norm $\nu$ on $C^{n}$ as described in statement (ii) of Proposition 1.

Proposition 9. Let $C^{n}=X_{1} \oplus \cdots \oplus X_{k}$ be a direct-sum decomposition of $C^{n}$, let $E_{1}, \cdots, E_{k}$ be the associated projections, let $\nu: C^{n}$ $\rightarrow R_{+}^{k}$ be the vectorial norm defined by 


$$
p(x)=\left(\nu\left(E_{1} x\right), \cdots, \nu\left(E_{k} x\right)\right) \quad\left(x \in C^{n}\right) .
$$

If $\operatorname{lub}_{\nu} E_{j}=1(j=1, \cdots, k)$, then

$$
p^{D}(y)=\left(\nu^{D}\left(E_{1}^{*} y\right), \cdots, \nu^{D}\left(E_{k}^{*} y\right)\right) \quad\left(y \in C^{n}\right) .
$$

(Here $\nu^{D}$ denotes the dual norm of $\nu$ and lub $_{\nu}$ is the matrix norm subordinate to $\nu[3]$.)

Proof. We have, for all $y \in C^{n}$ and all $j=1, \cdots, k$,

$$
\begin{aligned}
p_{j}^{D}(y) & =\operatorname{Sup}_{x \in X_{j} ; x \neq 0} \frac{\left|y^{*} x\right|}{\nu\left(E_{j} x\right)}=\operatorname{Sup}_{x \in X_{j} ; x \neq 0} \frac{\left|y^{*} E_{j} x\right|}{\nu(x)} \leqq \operatorname{Sup}_{x \in C^{n} ; x \neq 0} \frac{\left|\left(E_{j}^{*} y\right)^{*} x\right|}{\nu(x)} \\
& =\nu^{D}\left(E_{j}^{*} y\right) .
\end{aligned}
$$

It is known that for every $y \in C^{n}$, there exists a nonzero $x_{0} \in C^{n}$ such that $\nu^{D}\left(E_{j}^{*} y\right) \nu\left(x_{0}\right)=\left|y^{*} E_{j} x_{0}\right|$. Then, taking into account that the relation $\operatorname{lub}_{\nu} E_{j}=1$ implies $\nu\left(E_{j} x_{0}\right) \leqq \nu\left(x_{0}\right)$, we have

$$
\nu^{D}\left(E_{j}^{*} y\right)=\frac{\left|y^{*} E_{j} x_{0}\right|}{\nu\left(x_{0}\right)} \leqq \frac{\left|y^{*} E_{j} x_{0}\right|}{\nu\left(E_{j} x_{0}\right)} \leqq \operatorname{Sup}_{x \in X_{j} ; x \neq 0} \frac{\left|y^{*} x\right|}{\nu(x)}=p_{j}^{D}(y) .
$$

Thus $p_{j}^{D}(y)=\nu^{D}\left(E_{j}^{*} y\right)$ for all $y \in C^{n}$ and all $j=1, \cdots, k$.

4. The $G$-transform of a vectorial pseudonorm. Some of the results of this section will be generalizations of the $G$-transform of a vector norm [2]. If $p$ is a vectorial pseudonorm of order $k$ on $C^{n}$, and $G$ is a complex $n \times n$ matrix, then it is easy to see that the mapping

$$
p_{G}: C^{n} \rightarrow R_{+}^{k}, \quad p_{G}(x)=p(G x) \quad\left(x \in C^{n}\right),
$$

is also a vectorial pseudonorm. The mapping $p_{G}$ will be called the $G$ transform of $p$.

Proposition 10. If $p$ is a vectorial norm, and $G$ is a nonsingular complex $n \times n$ matrix, then

(a) $p_{G}$ is also a vectorial norm;

(b) $K_{j}\left(p_{G}\right)=G^{-1} K_{j}(p)(j=1, \cdots, k)$;

(c) $W_{j}\left(p_{G}\right)=G^{-1} W_{j}(p)(j=1, \cdots, k)$;

(d) $W\left(p_{G}\right)=G^{-1} W(p)$;

(e) if $p$ is regular, then $p_{G}$ is also regular;

(f) the dual of $p_{G}$ is the $\left(G^{-1}\right)^{*}$-transform of the dual of $p$;

(g) if $G$ is unitary, then the dual of the G-transform of $p$ is equal to the $G$-transform of the dual of $p$.

Proof. (a) Let $x \in C^{n}$ such that $p_{G}(x)=0$. Then $p(G x)=0$ whence $G x=0$ and so $x=G^{-1} G x=0$. 
(b) $K_{j}\left(p_{G}\right)=\left\{x \in C^{n}: p_{j}(G x)=0\right\}=\left\{x \in C^{n}: G x \in K_{j}(p)\right\}=G^{-1} K_{j}(p)$ $(j=1, \cdots, k)$.

(c) Since $G$ is nonsingular, we have $W_{j}\left(p_{G}\right)=\bigcap_{h \neq j} K_{h}\left(p_{G}\right)$ $=\bigcap_{h \neq j} G^{-1} K_{h}(p)=G^{-1} \bigcap_{h \neq j} K_{h}(p)=G^{-1} W_{j}(p)(j=1, \cdots, k)$.

(d) $W\left(p_{G}\right)=\sum_{h} W_{h}\left(p_{G}\right)=\sum_{h} G^{-1} W_{h}(p)=G^{-1} W(p)$.

(e) This is an immediate consequence of (d).

(f) Making use of (c) we have, for all $j=1, \cdots, k$ and for all $y \in C^{n}$,

$$
\begin{aligned}
\left(p_{G}\right)_{j}^{D}(y) & =\operatorname{Sup}_{x \in G^{-1}} \frac{\left|y^{*} x\right|}{W_{j}(p) ; x \neq 0}=\operatorname{Sup}_{p_{j}(G x)} \frac{\left|y^{*} G^{-1} z\right|}{p_{j}(z)} \\
& =p_{j}^{D}\left(G^{-1^{*}} y\right)=\left(\left(p^{D}\right)_{G^{-1^{*}}}\right)_{j}(y),
\end{aligned}
$$

whence $\left(p_{G}\right)^{D}=\left(p^{D}\right)_{G^{-1^{*}}}$.

(g) This follows from (f).

\section{REFERENCES}

1. M. Fiedler and V. Pták, Generalized norms of matrices and the location of the spectrum, Czechoslovak Math. J. 12 (87) (1962), 558-571. MR 26 \#4187.

2. A. S. Householder, The approximate solution of matrix problems, J. Assoc. Comput. Mach. 5 (1958), 205-243. MR 23 \#B1646.

3. - The theory of matrices in numerical analysis, Blaisdell, Waltham, Mass., 1964. MR $30 \# 5475$.

4. L. V. Kantorovix, The method of successive approximations for functional equations, Acta Math. 71 (1939), 63-97. MR 1, 18.

5. L. V. Kantorovič, B. Z. Vulih and A. G. Pinsker, Functional analysis in partially ordered spaces, GITTL, Moscow, 1950. (Russian) MR 12, 340.

6. - Partially ordered groups and partially ordered linear spaces, Uspehi Mat. Nauk 6 (1951), no. 3 (43), 31-98; English transl., Amer. Math. Soc. Transl. (2) 27 (1963), 51-124. MR 13, 361; MR 27 \#1517.

7. A. M. Ostrowski, On some metrical properties of operator matrices and matrices partitioned into blocks, J. Math. Anal. Appl. 2 (1961), 161-209. MR 24 \#A421.

8. - Iterative solution of linear systems of functional equations, J. Math. Anal. Appl. 2 (1961), 351-369. MR 23 \#B1672.

9. F. Robert, Normes vectorielles de vecteurs et de matrices, Rev. Française Traitement Information Chiffres 7 (1964), 261-299. MR 34 \#5261.

10. - Sur les normes vectorielles régulières sur un espace vectoriel de dimension finie, C. R. Acad. Sci. Paris 261 (1965), 5173-5176. MR 33 \#543.

11. - Recherche d'une M-matrice parmi les minorantes d'un opérateur linéaire, Numer. Math. 9 (1966), 189-199. MR 34 \#8630.

12. J. Stoer, Lower bounds of matrices, Numer. Math. 12 (1968), 146-158. MR $38 \# 5378$.

Polytechnic Institute of Brooklyn, Brooklyn, New York 11201 